\title{
Signum Function Method for Generation of Correlated Dichotomic Chains
}

\author{
S. S. Apostolov, ${ }^{1}$ F. M. Izrailev, ${ }^{2, \text {, }}$ N. M. Makarov, ${ }^{1, \text { I }}$ Z. A. Mayzelis, ${ }^{1}$ S. S. Melnyk, ${ }^{1}$ and O. V. Usatenko ${ }^{1}$, 团 \\ ${ }^{1}$ A. Ya. Usikov Institute for Radiophysics and Electronics, \\ Ukrainian Academy of Science, 12 Proskura Street, 61085 Kharkov, Ukraine \\ ${ }^{2}$ Instituto de Física, Universidad Autónoma de Puebla, \\ Apartado Postal J-48, Puebla, Pue., 72570, México
}

(Dated: November 11, 2018)

\begin{abstract}
We analyze the signum-generation method for creating random dichotomic sequences with prescribed correlation properties. The method is based on a binary mapping of the convolution of continuous random numbers with some function originated from the Fourier transform of a binary correlator. The goal of our study is to reveal conditions under which one can construct binary sequences with a given pair correlator. Our results can be used in the construction of superlattices and waveguides with selective transport properties.

PACS numbers: 05.40.2a, 02.50.Ga, 87.10.1e
\end{abstract}

\section{INTRODUCTION}

The study of properties of disordered complex systems with spatial and/or temporal correlations is one of the hot topics in modern physics. Recently, much attention was paid to the related problem of how to construct disordered materials with specific transport properties that are due to underlying correlations in a disorder. One of the important applications of this problem is a creation of electron nano-devices, optic fibers, rough surfaces, acoustic and electromagnetic waveguides with selective transport properties.

It is known (see, for instance, [1, 2, 3, 4] ) that many of properties of systems with weak disorder are determined by the binary (pair or two-point) correlation function of a corresponding random process. Recently it was found [5, 6, 7] that specific long-range correlations in disordered potentials can lead to anomalous transport properties. To date, there exist a number of algorithms for generating long-range correlated sequences with prescribed correlations. Among such algorithms the most widespread one is the convolution method [6, 7, 8, 9, 10, 11, 12, 13, 14, 15, 16]. In this method random elements in the generated chain can be of any value from $-\infty$ to $\infty$. However, in many applications it is more convenient to construct the sequences with finite number of random values. An important example of such system, being occurred in nature, is a sequence of nucleotides in a DNA molecule, consisting of four elements only.

The simplest case of a random sequence of finite elements is a stochastic dichotomic (binary) chain of only

\footnotetext{
*Electronic address: izrailev@venus.ifuap.buap.mx

$\dagger^{\dagger}$ Electronic address: makarov@siu.buap.mx; On sabbatical leave from Instituto de Ciencias, Universidad Autónoma de Puebla, Priv. 17 Norte No. 3417, Col. San Miguel Hueyotlipan, Puebla, Pue., 72050, México.

${ }^{\ddagger}$ Electronic address: usatenko@ire.kharkov.ua
}

two elements. In contrast with the case of sequences of continuous elements, the problem of a construction of binary sequences with given correlation properties turns out to be tricky. As was recently shown in Ref. [16], there is a serious restriction on the type of pair correlators in binary sequences, in contrast with the sequences with continuous distribution of their elements. Many of related results for binary sequences can be found in Refs. 17, 18, 19.

A direct way to create dichotomic correlated sequences is to apply the signum function to the sequence of continuous values, obtained with the convolution method. This method is based on the convolution of a whitenoise sequence with some function that is determined by the desired pair correlator. However, as was numerically found in [20], the created binary sequence turns out to have the pair correlator different from the expected one. To date, it remains unclear how to construct binary sequences having the same pair correlators as in the sequences with continuous values of their elements. In spite of its quite simple form, the signum function method is not rigorously analyzed in the literature.

The present paper makes an effort to clarify this problem. Our aim is to understand under what conditions the discussed method allows to construct binary sequences with a desired pair correlator. We perform a detailed theoretical analysis of the restrictions arising for binary sequences with long-range correlations. Main attention is paid to the step-wise power spectrum resulting in a power decay of correlations. This type of correlations is extremely important in various applications, such as a creation of devices with selective transport properties.

The paper is organized as follows. Section 2 is devoted to general properties of binary sequences. In particular, we derive some of the conditions restricting the form of pair correlators that a binary sequence can have. In Section 3 we describe in details the signum-generation method and derive basic relations needed for a further analysis. In next Section 4 we analyze the case of a balanced (unbiased) dichotomic sequence, i.e., the sequence with the zero mean-value. Here we also display the re- 
strictions related to the discussed method. Our main findings are reported in Section 5 where we consider the most interesting case of long-range correlations with the step-wise spectrum. In Section 6 we ask a question about general restrictions appearing in the case of power decay of correlations for binary sequences. In last Section we give some additional remarks concerning binary sequences, and summarize our results. The Appendices contain some of details of analytical and numerical calculations.

\section{NECESSARY CONDITIONS}

Let us start with generic properties of dichotomic sequences, not associated with specific choice of a generation method. In view of constructing the sequences with a given pair correlator, it is of great importance to know what are restrictions on the type of correlators allowed for dichotomic sequences. To shed light on this problem, here we consider a statistically homogeneous random sequence of symbols $s_{n}$ consisting of the values " -1 " and "1",

$$
s_{n}=\{-1,1\}, \quad n \in \mathbf{Z}=\ldots,-2,-1,0,1,2, \ldots
$$

The canonical definition of the correlation function reads as follows

$$
C_{s}(r) \equiv \overline{s_{n} s_{n+r}}-\bar{s}^{2}=C_{s}(0) K_{s}(r),
$$

where $\bar{s} \equiv \overline{s_{n}}$ and $C_{s}(0) \equiv \overline{s_{n}^{2}}-\bar{s}^{2}$ are the mean value and variance of $s_{n}$, respectively. Taking into account a peculiar property

$$
\overline{s_{n}^{2}}=s_{n}^{2}=1
$$

of our dichotomic sequence $s_{n}$, one obtains

$$
C_{s}(0)=1-\bar{s}^{2} \text {. }
$$

It should be emphasized that the direct relation between the mean value and higher one-point moments is a specific property of any dichotomic chain. For instance, for the binary chain $\varepsilon_{n}$ consisting of " 0 " and " 1 " we have the relation $C_{\varepsilon}(0)=\bar{\varepsilon}(1-\bar{\varepsilon})$ since $\varepsilon_{n}^{2}=\varepsilon_{n}$ in this case. On the contrary, for a sequence of continuous random numbers of the Gaussian type the variance and mean value are independent parameters .

To proceed with the two-point moments, we associate the correlator $C_{s}(r)$ with the probabilities of two symbols with the same or opposite signs, occurring at the distance $r$,

$$
\begin{aligned}
& 4 P( \pm 1, \underbrace{\ldots}_{r-1}, \pm 1)=(\bar{s} \pm 1)^{2}+C_{s}(r), \\
& 4 P( \pm 1, \underbrace{\ldots}_{r-1}, \mp 1)=1-\bar{s}^{2}-C_{s}(r) .
\end{aligned}
$$

These relationships also are peculiar properties solely of dichotomic sequences, they are drastically distinct from the analogous relations for other random processes (see, e.g., Eq. (B8) for the two-point probability density of the Gaussian process). This fact is strictly confirmed by straightforward calculations of Eqs. (5).

In order to obtain the expression (5a), one should write the average $\overline{\left(s_{n} \pm 1\right)\left(s_{n+r} \pm 1\right)}$ via the correlator (2),

$$
\begin{aligned}
\overline{\left(s_{n} \pm 1\right)\left(s_{n+r} \pm 1\right)} & =\overline{s_{n} s_{n+r}} \pm \overline{s_{n}} \pm \overline{s_{n+r}}+1 \\
& =C_{s}(r)+(\bar{s} \pm 1)^{2} .
\end{aligned}
$$

On the other hand, the same average can be calculated with the use of two-symbol probabilities,

$$
\begin{aligned}
& \overline{\left(s_{n}+1\right)\left(s_{n+r}+1\right)}=2 \cdot 2 \cdot P(1, \underbrace{\ldots}_{r-1}, 1) \\
& +0 \cdot 2 \cdot P(-1, \underbrace{\ldots}_{r-1}, 1)+2 \cdot 0 \cdot P(1, \underbrace{\ldots}_{r-1},-1) \\
& +0 \cdot 0 \cdot P(-1, \underbrace{\ldots}_{r-1},-1)=4 P(1, \underbrace{\ldots}_{r-1}, 1) . \\
& \overline{\left(s_{n}-1\right)\left(s_{n+r}-1\right)}=0 \cdot 0 \cdot P(1, \underbrace{\ldots}_{r-1}, 1) \\
& +(-2) \cdot 0 \cdot P(-1, \underbrace{\ldots}_{r-1}, 1)+0 \cdot(-2) \cdot P(1, \underbrace{\ldots}_{r-1},-1) \\
& +(-2) \cdot(-2) \cdot P(-1, \underbrace{\ldots}_{r-1},-1)=4 P(-1, \underbrace{\ldots}_{r-1},-1) \text {. }
\end{aligned}
$$

Then, the combination of Eqs. (6), (7), and (8) results in the equality (5a).

Similarly, in order to derive the expression (5b), one can write,

$$
\begin{aligned}
\overline{\left(s_{n} \pm 1\right)\left(s_{n+r} \mp 1\right)} & =\overline{s_{n} s_{n+r}} \mp \overline{s_{n}} \pm \overline{s_{n+r}}-1 \\
& =C_{s}(r)+\bar{s}^{2}-1 .
\end{aligned}
$$

Again, the above average can be calculated employing two-symbol probabilities. Specifically,

$$
\begin{array}{r}
\overline{\left(s_{n}+1\right)\left(s_{n+r}-1\right)}=2 \cdot 0 \cdot P(1, \underbrace{\ldots}_{r-1}, 1) \\
+0 \cdot 0 \cdot P(-1, \underbrace{\ldots}_{r-1}, 1)+2 \cdot(-2) \cdot P(1, \underbrace{\ldots}_{r-1},-1) \\
+0 \cdot(-2) \cdot P(-1, \underbrace{\ldots}_{r-1},-1)=-4 P(1, \underbrace{\ldots,}_{r-1},-1) . \\
+(-2) \cdot 2 \cdot P(-1, \underbrace{\ldots}_{r-1}, 1)+0 \cdot 0 \cdot P(1, \underbrace{\ldots}_{r-1},-1) \\
+(-2) \cdot 0 \cdot P(-1, \underbrace{\ldots}_{r-1},-1)=-4 P(-1, \underbrace{\ldots \ldots}_{r-1}, 1) .
\end{array}
$$


From Eqs. (9), (10), and (11) it follows the equality (5b).

Now, with the use of expressions (5a) we express the correlation function $C_{s}(r)$ via the probabilities to occur three symbols,

$$
C_{s}(r)+(\bar{s} \pm 1)^{2}=4 \sum_{a=-1,1} P( \pm 1, \underbrace{\ldots}_{r^{\prime}-1}, a, \underbrace{\ldots}_{r-r^{\prime}-1}, \pm 1) .
$$

Probability $P( \pm 1, \underbrace{\ldots}_{r^{\prime}-1}, a, \underbrace{\ldots}_{r-r^{\prime}-1}, \pm 1)$ is smaller than both probabilities $P( \pm 1, \underbrace{\ldots}_{r^{\prime}-1}, a)$ and $P(a, \underbrace{\ldots}_{r-r^{\prime}-1}, \pm 1)$. Thus, we can write,

$$
\begin{aligned}
& C_{s}(r)+(\bar{s} \pm 1)^{2} \\
& \leqslant 4 \sum_{a=-1,1} \min \left\{P( \pm 1, \underbrace{\ldots}_{r^{\prime}-1}, a), P(a, \underbrace{\ldots}_{r-r^{\prime}-1}, \pm 1)\right\} \\
& =\min \left\{1-\bar{s}^{2}-C_{s}\left(r^{\prime}\right), 1-\bar{s}^{2}-C_{s}\left(r-r^{\prime}\right)\right\} \\
& +\min \left\{(\bar{s} \pm 1)^{2}+C_{s}\left(r^{\prime}\right),(\bar{s} \pm 1)^{2}+C_{s}\left(r-r^{\prime}\right)\right\} \\
& =1-\bar{s}^{2}+\min \left\{-C_{s}\left(r^{\prime}\right),-C_{s}\left(r-r^{\prime}\right)\right\} \\
& +(\bar{s} \pm 1)^{2}+\min \left\{C_{s}\left(r^{\prime}\right), C_{s}\left(r-r^{\prime}\right)\right\} .
\end{aligned}
$$

Here we again have used Eqs. (5). Then, according to the evident relation

$$
\min \{x, y\}+\min \{-x,-y\}=-|x-y|,
$$

we arrive at the condition

$$
\left|C_{s}\left(r^{\prime}\right)-C_{s}\left(r-r^{\prime}\right)\right|+C_{s}(r) \leqslant 1-\bar{s}^{2} .
$$

Finally, it is convenient to rewrite Eq. (15) for the normalized correlator $K_{s}(r)$,

$$
\left|K_{s}\left(r^{\prime}\right)-K_{s}\left(r-r^{\prime}\right)\right|+K_{s}(r) \leqslant 1 .
$$

Although we have derived this inequality for $0<r^{\prime}<r$, a simple analysis reveals its validity for arbitrary values of $r$ and $r^{\prime}$. We would like to stress that the condition (16) is applicable for any mean value $\bar{s}$ of the dichotomic sequence $s_{n}$. However, without a loss of generality in what follows we consider binary sequences with the zero mean, since the statistical properties of considered sequences do not depend on mean values.

In a similar manner one can obtain second inequality with the use of Eq. (5b),

$$
\begin{aligned}
& 1-\bar{s}^{2}-C_{s}(r)=4 \sum_{a=-1,1} P( \pm 1, \underbrace{\ldots}_{r^{\prime}-1}, a, \underbrace{\ldots}_{r-r^{\prime}-1}, \mp 1) \\
& \leqslant 4 \sum_{a=-1,1} \min \left\{P( \pm 1 \underbrace{\ldots}_{r^{\prime}-1}, a), P(a, \underbrace{\ldots}_{r-r^{\prime}-1}, \mp 1)\right\} \\
& =\min \left\{1-\bar{s}^{2}-C_{s}\left(r^{\prime}\right),(\bar{s} \mp 1)^{2}+C_{s}\left(r-r^{\prime}\right)\right\} \\
& +\min \left\{(\bar{s} \pm 1)^{2}+C_{s}\left(r^{\prime}\right), 1-\bar{s}^{2}-C_{s}\left(r-r^{\prime}\right)\right\} .
\end{aligned}
$$

Since $C_{s}(r)=K_{s}(r)$ in the case of $\bar{s}=0$, Eq. (17) gets a simpler form,

$$
\left|K_{s}\left(r^{\prime}\right)+K_{s}\left(r-r^{\prime}\right)\right|-K_{s}(r) \leqslant 1 \quad \text { for } \quad \bar{s}=0 .
$$

Thus, applying the necessary conditions (16) and (17), or (18) if $\bar{s}=0$, one can identify the functions that can not be treated as binary correlators of a dichotomic sequence.

Inequalities (16), (17) and (18) have to be met for any values of indices $r$ and $r^{\prime}$ and, therefore, they actually represent an infinite set of necessary conditions. Evidently, in every particular case one should choose the strongest condition. On the other hand, Eqs. (16), (17) and (18) are automatically satisfied if one of the indices equals zero, $r=0$ or $r^{\prime}=0$. The same takes place when $r=r^{\prime}$. Summarizing all these facts, we can combine Eqs. (16) and (18) as follows

$$
\begin{aligned}
& \max \left\{\left|K_{s}\left(r^{\prime}\right) \pm K_{s}\left(r-r^{\prime}\right)\right| \mp K_{s}(r)\right\} \leqslant 1 \\
& r \neq 0, \quad r^{\prime} \neq 0, \quad r \neq r^{\prime} \quad \text { for } \quad \bar{s}=0 .
\end{aligned}
$$

The symbol $\max \{\ldots\}$ implies the absolute maximum of a function with respect to the indices $r, r^{\prime}$. Note that Eq. (19) is automatically fulfilled for two limit cases, namely, for the delta-correlated (white noise) chain, and for the sequences with infinitely long-range correlations. As one can see, for both these cases $K_{s}(r)=\delta_{r, 0}$ or $K_{s}(r)=1$, respectively.

\section{BINARY VERSUS GAUSSIAN}

Now we analyze the construction of a dichotomic sequence $\gamma_{n}$ by means of the signum function generation (SFG) method. It uses an intermediate correlated disorder $\beta_{n}$ obtained as a convolution of the uncorrelated Gaussian noise $\alpha_{n}$ and modulation function $G(n)$. Specifically, the $\gamma$-sequence is defined by

$$
\begin{aligned}
& \gamma_{n}=\operatorname{sign}\left(\beta_{n}\right) \\
& \beta_{n}=\bar{\beta}+\sum_{n^{\prime}=-\infty}^{\infty} G\left(n-n^{\prime}\right) \alpha_{n^{\prime}}
\end{aligned}
$$

The initial Gaussian white-noise chain consists of stochastic variables $\alpha_{n}$ with the zero mean, unit variance and corresponding probability density. Respectively,

$$
\begin{aligned}
& \bar{\alpha}=0, \quad \overline{\alpha_{n} \alpha_{n^{\prime}}}=\delta_{n, n^{\prime}}, \\
& \rho_{A}\left(\alpha_{n}=\alpha\right)=\frac{1}{\sqrt{2 \pi}} \exp \left(-\alpha^{2} / 2\right) .
\end{aligned}
$$

The bar over a random symbol or function implies the stochastic average.

Evidently, the constructed dichotomic sequence $\gamma_{n}$ does not change if one normalizes the intermediate sequence $\beta_{n}$ by an arbitrary factor. Hence, without any loss of generality, we can admit the variance of $\beta_{n}$ be 
equal to unity. Then, $\bar{\beta}$ is the mean value of $\beta_{n}$ and its correlation function $K_{\beta}(r)$,

$$
K_{\beta}(r)=\overline{\left(\beta_{n}-\bar{\beta}\right)\left(\beta_{n+r}-\bar{\beta}\right)},
$$

is normalized to unity, $K_{\beta}(0)=1$. By direct substitution of Eq. (20b) into definition (22), the correlator $K_{\beta}(r)$ is readily associated with the modulation function $G(n)$,

$$
K_{\beta}(r)=\sum_{n=-\infty}^{\infty} G(r-n) G(n) .
$$

Owing to evenness of $K_{\beta}(r)=K_{\beta}(-r)$ and in accordance with Eq. (23), one can also restrict the function $G(n)$ to the class of even functions, $G(-n)=G(n)$. Note that the condition $K_{\beta}(0)=1$ gives rise to the following normalization for $G(n)$,

$$
K_{\beta}(0)=\sum_{n=-\infty}^{\infty} G^{2}(n)=1 .
$$

It is convenient to pass to the Fourier transform of Eq. (23) with the use of the standard expressions,

$$
\begin{aligned}
& K_{\beta}(r)=\frac{1}{2 \pi} \int_{-\pi}^{\pi} d k \mathcal{K}_{\beta}(k) \exp (i k r) \\
& \mathcal{K}_{\beta}(k)=\sum_{r=-\infty}^{\infty} K_{\beta}(r) \exp (-i k r)
\end{aligned}
$$

The Fourier transform $\mathcal{K}_{\beta}(k)$ of the pair correlator $K_{\beta}(r)$ introduced here, is known as the power spectrum of ran$\operatorname{dom} \beta$-chain. Since the correlator $K_{\beta}(r)$ is real and even function of $r$, the power spectrum (25b) is real, even, $\mathcal{K}_{\beta}(-k)=\mathcal{K}_{\beta}(k)$, and non-negative function of the wave number $k$. Analogously to Eq. (25), for the modulation function $G(n)$ one can define its Fourier transform $\mathcal{G}(k)$, which is also real and even function, $\mathcal{G}(-k)=\mathcal{G}(k)$.

The Fourier representation of Eq. (23) reads

$$
\mathcal{K}_{\beta}(k)=\mathcal{G}^{2}(k) .
$$

Thus, we arrive at the following expression for the modulation function $G(n)$,

$$
G(n)=\frac{1}{\pi} \int_{0}^{\pi} d k \mathcal{K}_{\beta}^{1 / 2}(k) \cos (k n) .
$$

Evidently, the solution (27) automatically satisfies the normalization condition (24).

Since the initial chain $\alpha_{n}$ is a delta-correlated Gaussian noise, the intermediate variables $\beta_{n}$ also constitute a Gaussian random sequence with single-point distribution

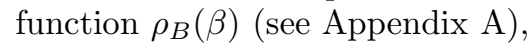

$$
\rho_{B}\left(\beta_{n}=\beta\right)=\frac{1}{\sqrt{2 \pi}} \exp \left[-(\beta-\bar{\beta})^{2} / 2\right] .
$$

In order to reveal statistical properties of the signumgenerated dichotomic sequence $\gamma_{n}$, one should associate its mean value $\bar{\gamma}$, variance $C_{\gamma}(0)$ and pair correlator $C_{\gamma}(r)$ with the corresponding independent characteristics, namely, the mean value $\bar{\beta}$ and the modulation function $G(n)$ [or, the same, with the intermediate correlator $\left.K_{\beta}(r)\right]$. According to the definition of average, one can express the mean value $\bar{\gamma}$ in terms of $\bar{\beta}$ via the error function [21],

$$
\begin{aligned}
\bar{\gamma} \equiv \overline{\gamma_{n}} & =\int_{-\infty}^{\infty} d \beta \rho_{B}(\beta) \operatorname{sign}(\beta) \\
& =\sqrt{\frac{2}{\pi}} \int_{0}^{\bar{\beta}} d x \exp \left(-x^{2} / 2\right) \equiv \operatorname{erf}(\bar{\beta} / \sqrt{2}) .
\end{aligned}
$$

Similarly to Eq. (4), the variance $C_{\gamma}(0)$ is written in the form

$$
C_{\gamma}(0) \equiv \overline{\gamma_{n}^{2}}-\bar{\gamma}^{2}=1-\bar{\gamma}^{2} .
$$

An important characteristic of the stochastic sequence $\gamma_{n}$ is the correlation function $C_{\gamma}(r)$,

$$
C_{\gamma}(r) \equiv \overline{\gamma_{n} \gamma_{n+r}}-\bar{\gamma}^{2}=C_{\gamma}(0) K_{\gamma}(r) .
$$

Its calculation is performed in Appendix B] Here we write down only the final equation that relates the correlator $K_{\gamma}(r)$ to $K_{\beta}(r)$,

$$
\begin{aligned}
(1 & \left.-\bar{\gamma}^{2}\right) K_{\gamma}(r) \\
& =\frac{2}{\pi} \int_{0}^{K_{\beta}(r)} \frac{d x}{\sqrt{1-x^{2}}} \exp \left(-\frac{\bar{\beta}^{2}}{1+x}\right) .
\end{aligned}
$$

Note that r.h.s. of the latter equation is not elementary function, therefore its analytical study is not simple. However, the case $\bar{\beta}=0$ allows one to perform complete analytical analysis.

\section{UNBIASED SEQUENCE}

If the mean value of intermediate chain $\beta_{n}$ vanishes, $\bar{\beta}=0$, then due to Eq. (29), the mean value of generated sequence $\gamma_{n}$ vanishes also, $\bar{\gamma}=0$. In this case the relation (32) turns out to be remarkably simplified,

$$
K_{\gamma}(r)=\frac{2}{\pi} \int_{0}^{K_{\beta}(r)} \frac{d x}{\sqrt{1-x^{2}}}=\frac{2}{\pi} \arcsin \left[K_{\beta}(r)\right] .
$$

Another equivalent form is

$$
K_{\beta}(r)=\sin \left[\frac{\pi}{2} K_{\gamma}(r)\right] .
$$

From the above relations one can conclude that the $\gamma$ sequence generated with the discussed signum function method, is random. Indeed, the decay of correlations with an increase of $|r|$ in the intermediate $\beta$-chain, also results in the decay of correlations in the generated dichotomic $\gamma$-sequence. 
Now it is suitable to rewrite Eq. (34) in the Fourier representation,

$$
\mathcal{K}_{\beta}(k)=\mathcal{S}\left\{K_{\gamma}\right\}(k) .
$$

Here the symbol $\mathcal{S}\{\cdot\}(k)$ stands for the operator that transforms the function $K(r)$ by the following rule,

$$
\begin{aligned}
& \mathcal{S}\{K\}(k) \equiv \sum_{r=-\infty}^{\infty} \sin \left[\frac{\pi}{2} K(r)\right] \exp (-i k r) \\
& =\left(1-\frac{\pi}{2}\right)+\frac{\pi}{2} \mathcal{K}(k) \\
& +2 \sum_{r=1}^{\infty}\left\{\sin \left[\frac{\pi}{2} K(r)\right]-\frac{\pi}{2} K(r)\right\} \cos (k r)
\end{aligned}
$$

It is important to note that the series (36a) can converge very slowly. Therefore, in the analytical and numerical analysis one has to take into account a lot of terms in the sum in order to obtain correct result. To avoid this problem, we have used the following trick that is based on the second equality (36b). Namely, since $K(r) \rightarrow 0$ when $|r| \rightarrow \infty$, the latter sum converges quite rapidly according to the asymptotic relation

$$
\sin \left[\frac{\pi}{2} K(r)\right]-\frac{\pi}{2} K(r) \rightarrow \frac{\pi^{3}}{48} K^{3}(r), \quad|r| \rightarrow \infty
$$

The substitution of Eq. (35) into Eq. (27) yields the following final relation between the modulation function $G(n)$ and the correlator $K_{\gamma}(r)$ of the generating dichotomic noise $\gamma_{n}$,

$$
G(n)=\frac{1}{\pi} \int_{0}^{\pi} d k \sqrt{\mathcal{S}\left\{K_{\gamma}\right\}(k)} \cos (k n) .
$$

Since the correlator $K_{\gamma}(r)$ is supposed to be known, the relation (38) should be regarded as the expression determining the modulation function $G(n)$.

As one can see, the SFG method for constructing the correlated dichotomic sequence $\gamma_{n}$ with the zero mean, unit variance and prescribed two-point correlator $K_{\gamma}(r)$ reduces to the following steps. First, starting from a desirable profile of $K_{\gamma}(r)$ and employing Eqs. (36) and (38), one has to obtain the modulation function $G(n)$. After, the correlated sequence $\gamma_{n}$ can be generated in accordance with Eq. (20). However, it is important to take into account the restriction that directly follows from Eq. (35). Specifically, since the power spectrum of any random process, in particular $\mathcal{K}_{\beta}(k)$, is a non-negative function of the wave number $k$, the function $\mathcal{S}\left\{K_{\gamma}\right\}(k)$ also has to be non-negative,

$$
\mathcal{S}\left\{K_{\gamma}\right\}(k) \geq 0 \quad \text { for } \quad|k| \leqslant \pi .
$$

This condition becomes apparent from Eq. (38), in which the function $\mathcal{S}\left\{K_{\gamma}\right\}(k)$ enters as a radicand. In other words, with this method the function $K_{\gamma}(r)$ can be considered as a correlator of a dichotomic random sequence $\gamma_{n}$, if and only if $\mathcal{S}\left\{K_{\gamma}\right\}(k)$ is a non-negative function of $k$.

In view of the revealed restriction (39), let us make a qualitative analysis of Eq. 36b . Note that the first summand $(1-\pi / 2)$ is negative. Therefore, if the spectrum $\mathcal{K}(k)$ vanishes within some interval of $k$ and the whole function $\mathcal{S}\{K\}(k)$ is non-negative there, consequently, the third summand is positive, exceeding the value $(\pi / 2-1)$. However, in the third summand only first few terms determine its sign and give correct estimate of the magnitude. Thus, one may expect that the correlation function of a dichotomic chain, which spectrum is zero in some interval, cannot be generated by the SFG method.

It should be stressed that the condition (39) is a quite serious restriction to the type of correlators allowed for dichotomic sequences. As a demonstration, let us consider simple example of the one-step additive Markov chain of variables $\varepsilon_{n}=\{0,1\}$ obtained according to the conditional probability

$$
P\left(\varepsilon_{n}=1 \mid \varepsilon_{n-1}\right)=\bar{\varepsilon}+\left(\varepsilon_{n-1}-\bar{\varepsilon}\right) \exp \left(-k_{c}\right),
$$

where the parameter $k_{c}$ is the inverse correlation length. As is known [22], this chain has an exponential pair correlator, $K_{\exp }(r)=\exp \left(-k_{c}|r|\right)$. It is instructive that the function $\mathcal{S}\left\{K_{\exp }\right\}(k)$ takes negative values for small $k_{c}$ below $k_{c}^{*} \approx 1.099 \ldots$, see the data in Fig. 1. Thus, the SFG method can reproduce exponential correlator only for $k_{c} \geqslant k_{c}^{*}$, however, not for small $k_{c}$ in the most interesting region of long-range correlations.

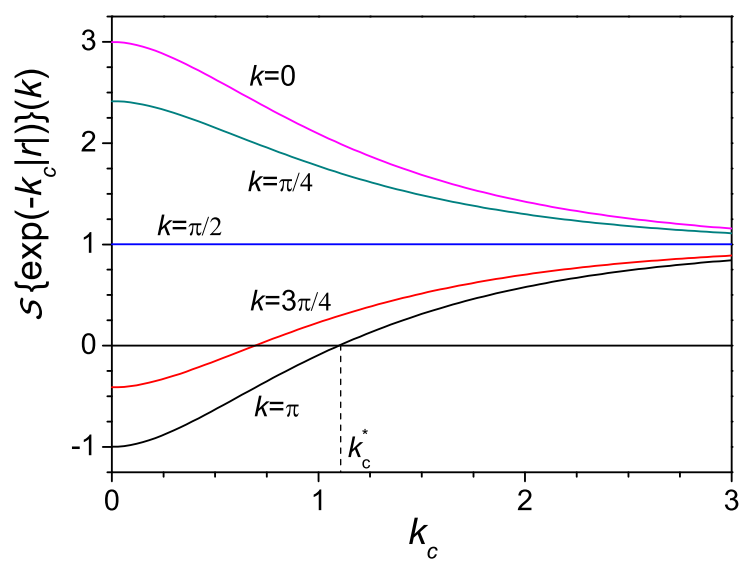

FIG. 1: (Color online) Dependence $\mathcal{S}\left\{\exp \left(-k_{c}|r|\right)\right\}(k)$ on the correlation parameter $k_{c}$ for several values of wave number $k$. 


\section{LONG-RANGE CORRELATORS WITH STEP-WISE SPECTRUM}

\section{A. Maximal Jump}

Here we demonstrate that the discussed method cannot be applied for a construction of dichotomic sequences with long-range correlators resulting in the step-wise power spectrum

$$
\begin{aligned}
& K_{\gamma}(r)=\frac{\sin \left(k_{c} r\right)}{k_{c} r}, \\
& \mathcal{K}_{\gamma}(k)=\frac{\pi}{k_{c}} \Theta\left(k_{c}-|k|\right), \quad 0<k_{c} \leqslant \pi, \quad|k| \leqslant
\end{aligned}
$$

This kind of correlations is of specific interest in view of applications to 1D disordered superlattices with a selective transport, see, e.g. [6, 7]. Here $k_{c}$ is the correlation parameter (inverse correlation length) to be specified, and $\Theta(x)$ implies the Heaviside unit-step function, $\Theta(x<0)=0$ and $\Theta(x>0)=1$.

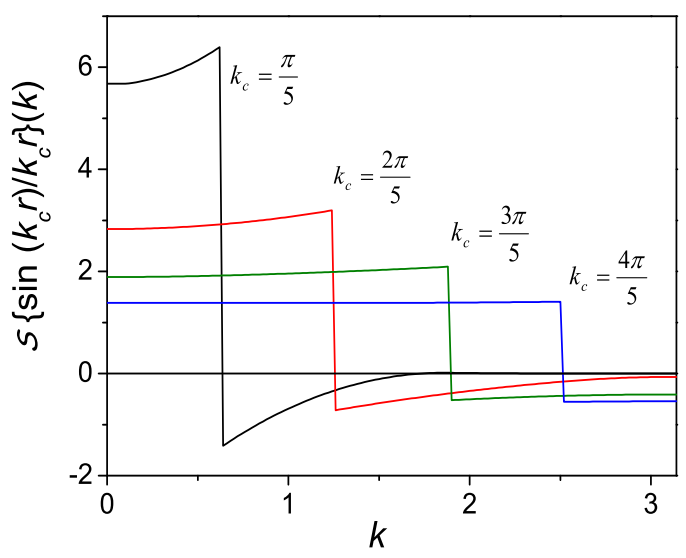

FIG. 2: (Color online) Dependence $\mathcal{S}\left\{\sin \left(k_{c} r\right) / k_{c} r\right\}(k)$ on $k$ for different values of $k_{c}$.

First, we analyze the values of $k_{c}$ within the interval $0<k_{c}<\pi$. Two specific cases of $k_{c}=0$ and $k_{c}=\pi$ will be considered afterwards.

In the analysis of the SFG method the crucial characteristic is the function $\mathcal{S}\left\{K_{\gamma}\right\}(k)$ defined by Eq. (36). It has to be non-negative for any value of the argument $k$ within the interval $|k| \leqslant \pi$, see Eq. (39). In the case of the long-range correlator (41a) it is suitable to use the following explicit expression for $\mathcal{S}\left\{K_{\gamma}\right\}(k)$,

$$
\begin{aligned}
& \mathcal{S}\left\{\frac{\sin \left(k_{c} r\right)}{k_{c} r}\right\}(k)=\left(1-\frac{\pi}{2}\right)+\frac{\pi^{2}}{2 k_{c}} \Theta\left(k_{c}-|k|\right) \\
& +2 \sum_{r=1}^{\infty}\left\{\sin \left[\frac{\pi}{2} \frac{\sin \left(k_{c} r\right)}{k_{c} r}\right]-\frac{\pi}{2} \frac{\sin \left(k_{c} r\right)}{k_{c} r}\right\} \cos (k r)(42)
\end{aligned}
$$

It is remarkable that the summand in the last term behaves as $\pi^{3} / 24 k_{c}^{3} r^{3}$ when $r \rightarrow \infty$. Hence, at finite $k_{c}$ the sum converges quite rapidly and uniformly. Therefore, the sum is a continuous function of $k$, in particular, at $k=k_{c}$, and can be easily calculated numerically.

In Fig. 22 the behavior of the radicand (42) in Eq. (38) is shown for several values of the inverse correlation length $k_{c}$. Since $\mathcal{S}\{\cdot\}(k)$ is an even function of the wave number $k$, the discussion can be restricted by the interval $0 \leqslant k \leqslant$ $\pi$. From Eq. (42) and Fig. 2 one can draw the following conclusions.

1. Due to the last term in expression (42) the function $\mathcal{S}\left\{\sin \left(k_{c} r\right) / k_{c} r\right\}(k)$ increases with an increase of $k$ for all $k_{c}$ within both intervals $\left(0, k_{c}\right)$ and $\left(k_{c}, \pi\right)$.

2. The negative jump of $\mathcal{S}\left\{\sin \left(k_{c} r\right) / k_{c} r\right\}(k)$ occurs at $k=k_{c}$, at the same point where the power spectrum (41b) has a jump. The maximal and minimal values of the function are achieved at $k=k_{c}-0$ and $k=k_{c}+0$, respectively. The jump is exclusively formed by the second term in Eq. (42). Therefore, its value reads

$$
\begin{aligned}
& \mathcal{S}\left\{\sin \left(k_{c} r\right) / k_{c} r\right\}\left(k=k_{c}-0\right) \\
& -\mathcal{S}\left\{\sin \left(k_{c} r\right) / k_{c} r\right\}\left(k=k_{c}+0\right)=\pi^{2} / 2 k_{c} .
\end{aligned}
$$

3. The minimal value $\mathcal{S}\left\{\sin \left(k_{c} r\right) / k_{c} r\right\}\left(k=k_{c}+0\right)$ is negative for all finite values of $k_{c}$ within the interval $0<k_{c}<\pi$. The complete dependence of positive function $-\mathcal{S}\left\{\sin \left(k_{c} r\right) / k_{c} r\right\}\left(k_{c}+0\right)$ on $k_{c}$ is depicted in Fig. 3

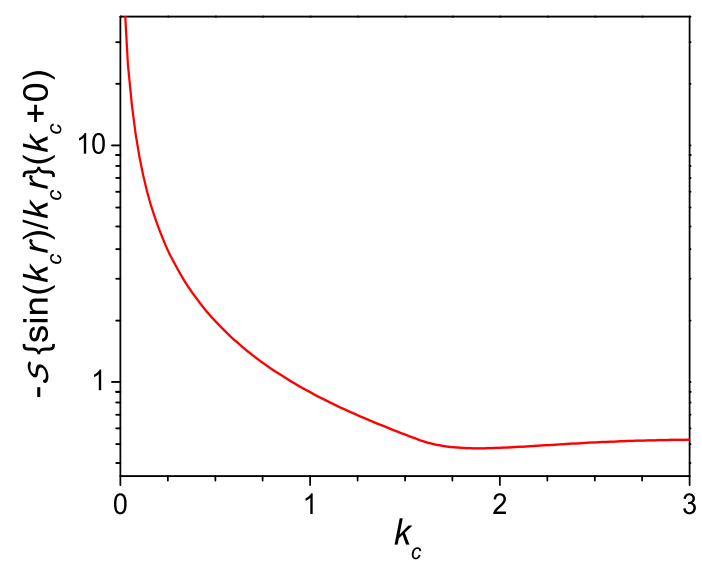

FIG. 3: (Color online) Dependence $-\mathcal{S}\left\{\sin \left(k_{c} r\right) / k_{c} r\right\}\left(k_{c}+0\right)$ vs $k_{c}$ in log-scale.

Item 3 displays clearly that for all finite values $k_{c}<\pi$ the correlator and corresponding power spectrum (41) cannot be created by making use of the discussed method. Indeed, the modulation function $G(n)$ turns out to be of complex value, see Eq. (38).

Now we determine the values of $k_{c}$ for which the function (41a) can not be the pair correlator of a dichotomic 
sequence regardless of the method of generation. This can be done with the use of the results of Sec. [II.

First, we demonstrate analytically that this function cannot be the correlation function of a dichotomic sequence $s_{n}$ with arbitrary mean value $\bar{s}$ for small but finite values of $k_{c}$, namely, for $0<k_{c} \ll 1$. To this end, we take Eq. (16) at $r^{\prime}=1$ and $r=r_{a}=\left[a / k_{c}\right]$, where $[x]$ is the integer part of $x$ and $a$ is a constant,

$$
\left|\frac{\sin k_{c}}{k_{c}}-\frac{\sin k_{c}\left(r_{a}-1\right)}{k_{c}\left(r_{a}-1\right)}\right|+\frac{\sin k_{c} r_{a}}{k_{c} r_{a}} \leqslant 1 .
$$

Being expanded in small parameter $k_{c}$, this condition reads

$$
\left(\frac{\cos a}{a}-\frac{\sin a}{a^{2}}\right) k_{c}+O\left(k_{c}^{2}\right) \leqslant 0 .
$$

It is evident that the l.h.s. of Eq. (45) can be positive at some values of $a$ (e.g., for $a=2 \pi+\pi / 4$ ). Thus, the requirement (16) is violated.

The inequality (18) can also result in new restrictions for allowed values of $k_{c}$ for the case $\bar{s}=0$. Rewriting it at $r=2$ and $r^{\prime}=1$, we get

$$
4 \sin k_{c}-\sin 2 k_{c} \leqslant 2 k_{c} \text {. }
$$

Numerical analysis shows that this condition does not hold true for all finite $k_{c}$ from the interval $0<k_{c}<k^{*}$, where $k^{*} \approx 2.139 \ldots$. It is met only at $k_{c}>k^{*}$. However, since Eq. (18) is just necessary condition, one cannot guarantee an existence of the correlator (41a) even at $k_{c}>k^{*}$.

At the critical point $k_{c}=\pi$ the correlator and spectrum (41) reduce to $K_{\gamma}(r)=\delta_{r, 0}$ and $\mathcal{K}_{\gamma}(k)=1$. This gives rise to the relations, $\mathcal{S}\left\{\delta_{r, 0}\right\}(k)=1$ and $G(n)=\delta_{n, 0}$, hence, $\beta_{n}=\alpha_{n}$. Consequently, one can apply the SFG. However, this specific case of $k_{c}=\pi$ is not interesting because from the Gaussian white-noise $\alpha_{n}$-sequence the method fabricates the dichotomic chain $\gamma_{n}$ again of white-noise type.

As to the singular point $k_{c}=0$, here we have $K_{\gamma}(r)=$ 1 for the correlator, and $\mathcal{K}_{\gamma}(k)=2 \pi \delta(k)$ for the power spectrum, therefore, the radicand is $\mathcal{S}\{1\}(k)=2 \pi \delta(k)$. One can see that the SFG is formally applicable. Besides, the necessary conditions (45) and (46) are satisfied automatically. However, this case is a singular one since for any arbitrarily small but finite values of $k_{c}$ it is not possible to create a dichotomic sequence with the correlation properties (41) neither by the SFG or by any other method.

\section{B. Partial Jump}

Now we extend our analysis to a more general correlation function that may have various applications. This function also results in a step-wise power spectrum, however, with an additional parameter $h$ that determines the height of step,

$$
\begin{aligned}
K_{\gamma, h}(r)= & h \delta_{r, 0}+(1-h) \frac{\sin \left(k_{c} r\right)}{k_{c} r}, \\
\mathcal{K}_{\gamma, h}(k)= & h+(1-h) \frac{\pi}{k_{c}} \Theta\left(k_{c}-|k|\right)>0, \\
& 0 \leqslant h \leqslant 1, \quad 0<k_{c} \leqslant \pi, \quad|k| \leqslant \pi .
\end{aligned}
$$

Eq. (47) coincides with Eq. (41) if the step-parameter $h=0$. Otherwise, when $h=1$ the generated $\gamma$-sequence turns into a dichotomic white noise independently of $k_{c}$. Also, $\gamma_{n}$ becomes delta-correlated at $k_{c}=\pi$ for arbitrary $h$. Therefore, at $k_{c}=\pi$ the conclusions of the previous subsection are also valid.

In what follows, it is convenient to analyze finite values of $k_{c}<\pi$. The power spectrum (47b) is an even function of the wave number $k$ and has two symmetric jumps at the points $k= \pm k_{c}$. For positive $0<k \leqslant \pi$ the spectrum abruptly falls down at $k=k_{c}$ from the maximal value $\mathcal{K}_{\gamma, h}\left(k<k_{c}\right)=h+(1-h) \pi / k_{c}$ to the minimal one, $\mathcal{K}_{\gamma, h}\left(k>k_{c}\right)=h$. Evidently, this jump can be regarded as a mobility edge of disordered 1D conductors, if the maximal value $h+(1-h) \pi / k_{c}$ is much larger than the minimal one, $h$,

$$
1+\frac{1-h}{h} \frac{\pi}{k_{c}} \gg 1 .
$$

One can see that for finite $h$ well above zero, this is possible only for small $k_{c}$. What is more tricky, for $k_{c} \ll 1$ the mobility edge may emerge even in the case when the generated $\gamma$-sequence is close to a dichotomic white noise, i.e., when $1-h \ll 1$. Therefore, one should have,

$$
0<k_{c} \ll 1-h \ll 1
$$

The reason of existence of the mobility edge under the conditions (49) is a significant contribution of the second term in the coorelator (47a). In spite of the fact that it has a quite small amplitude $1-h \ll 1$, this term provides extremely long-range correlations with the characteristic scale $k_{c}^{-1} \gg(1-h)^{-1} \gg 1$.

Now we address the function $\mathcal{S}\left\{K_{\gamma, h}\right\}(k)$ that must be non-negative in order to construct the correlated sequence $\gamma_{n}$ with the use of the SFG method. In accordance with the definition (36), an appropriate analysis can be done with the following explicit expression,

$$
\begin{aligned}
\mathcal{S}\left\{K_{\gamma, h}\right\}(k)= & 1-\frac{\pi}{2}(1-h)+(1-h) \frac{\pi^{2}}{2 k_{c}} \Theta\left(k_{c}-|k|\right) \\
& +2 \sum_{r=1}^{\infty}\left\{\sin \left[\frac{\pi}{2}(1-h) \frac{\sin \left(k_{c} r\right)}{k_{c} r}\right]\right. \\
& \left.-\frac{\pi}{2}(1-h) \frac{\sin \left(k_{c} r\right)}{k_{c} r}\right\} \cos (k r) .
\end{aligned}
$$

As in the previous case (41), the summand in the last term behaves as $\pi^{3} / 24 k_{c}^{3} r^{3}$ if $r \rightarrow \infty$. Hence, at finite $k_{c}$ 
the sum converges rapidly and uniformly. It is a continuous function of $k$, in particular, at $k=k_{c}$.

The numerical calculations of $\mathcal{S}\left\{K_{\gamma, h}(r)\right\}(k)$ performed for finite $0<k_{c}<\pi$ and $0 \leqslant k \leqslant \pi$, are shown in Fig. 4. Together with Eq. (50) they provide us with the following empirical results.

1. Due to the last term in Eq. (50), the function $\mathcal{S}\left\{K_{\gamma, h}\right\}(k)$ increases with $k$ at arbitrary values of $k_{c}$ and $h$ within both intervals $\left(0, k_{c}\right)$ and $\left(k_{c}, \pi\right)$.

2. The negative jump of $\mathcal{S}\left\{K_{\gamma, h}\right\}(k)$ occurs at the same point $k=k_{c}$ as for the jump of the prescribed power spectrum (47b). The maximal and minimal values of the function are reached, respectively, at $k=k_{c}-0$ and $k=k_{c}+0$ for all values of the step-parameter $h$ within $0 \leqslant h<1$. The jump is exclusively related to the third term in Eq. (50). Therefore, its value reads

$$
\mathcal{S}\left\{K_{\gamma, h}\right\}\left(k_{c}-0\right)-\mathcal{S}\left\{K_{\gamma, h}\right\}\left(k_{c}+0\right)=(1-h) \frac{\pi^{2}}{2 k_{c}} .
$$

3. Depending on $h$, the minimum $\mathcal{S}\left\{K_{\gamma, h}\right\}\left(k_{c}+0\right)$ can be either negative or positive. Also, the value of $\mathcal{S}\left\{K_{\gamma, h}\right\}\left(k_{c}+0\right)$ monotonically increases with an increase of $h$.

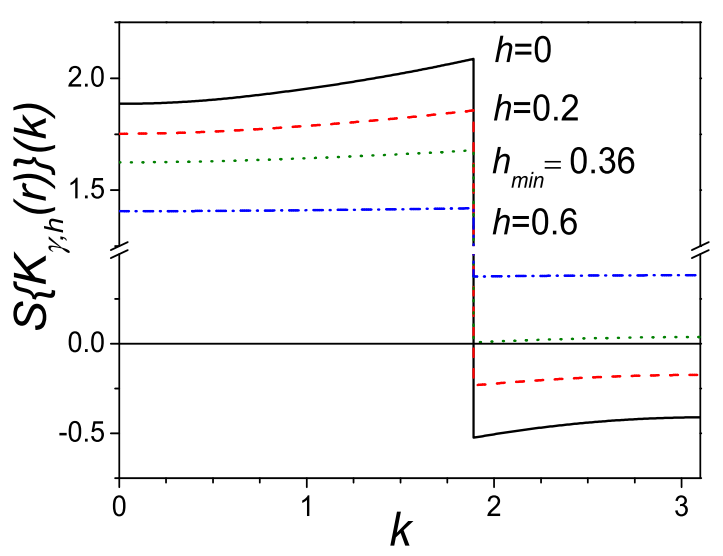

FIG. 4: (Color online) Function $\mathcal{S}\left\{K_{\gamma, h}(r)\right\}(k)$ vs $k$ for few values of $h$ at $k_{c}=1.89$ (this $k_{c}$ is very close to the minimum point of the curve in Fig. 3 and to the minimum point of the upper curve in Fig. [5). The function is entirely positive if $h>h_{\min }$. When $h=h_{\min }$, the function goes to zero solely at one point $k=k_{c}+0$ and is positive otherwise. For $h<h_{\min }$, the function is negative within the whole interval $k_{c}<k<\pi$.

The presented numerical analysis can be supplemented with the following two points. First, from the treatment of the case (41), we know that

$$
\mathcal{S}\left\{K_{\gamma, h}\right\}\left(k_{c}+0\right)<0 \quad \text { for } \quad h=0 .
$$

Second, from the definition (50) one can easily reveal that

$$
\mathcal{S}\left\{K_{\gamma, h}\right\}(k)=1 \quad \text { for } \quad h=1
$$

Summarizing our results, one can conclude that there exists a threshold value of the step-parameter $h$ that we refer to as $h_{\text {min }}$, that separates the region $0 \leqslant h<h_{\text {min }}$ in which $\mathcal{S}\left\{K_{\gamma, h}\right\}(k)$ has negative values, from the region with non-negative values,

$$
\mathcal{S}\left\{K_{\gamma, h}\right\}(k) \geqslant 0 \quad \text { for } \quad h_{\min } \leqslant h \leqslant 1 .
$$

It is clear that the threshold $h_{\text {min }}$ obeys the equation (see Fig. 4)

$$
\mathcal{S}\left\{K_{\gamma, h_{\min }}\right\}\left(k_{c}+0\right)=0,
$$

and depends on the correlation parameter $k_{c}$. By substitution of Eq. (50) into Eq. (55), one can rewrite it in explicit form,

$$
\begin{aligned}
& \frac{\pi}{4}\left(1-h_{\text {min }}\right)-\frac{1}{2}=\sum_{r=1}^{\infty}\left\{\sin \left[\frac{\pi}{2}\left(1-h_{\text {min }}\right) \frac{\sin \left(k_{c} r\right)}{k_{c} r}\right]\right. \\
& \left.-\frac{\pi}{2}\left(1-h_{\text {min }}\right) \frac{\sin \left(k_{c} r\right)}{k_{c} r}\right\} \cos \left(k_{c} r\right) .
\end{aligned}
$$

The numerical solution $h_{\min }\left(k_{c}\right)$ of this equation is displayed in Fig. 5 by the upper curve.

Eq. (56) can be solved analytically at small $k_{c}$, resulting in

$$
h_{\min }\left(k_{c}\right)=1-\left(\frac{96 k_{c}}{\pi^{4}}\right)^{1 / 3}+O\left(k_{c}^{2 / 3}\right) \quad \text { for } \quad k_{c} \ll 1 .
$$

This expression exhibits the limit $h_{\text {min }} \rightarrow 1$ for $k_{c} \rightarrow 0$.

Thus, in accordance with the study performed above, the dichotomic sequence $\gamma_{n}$ with long-range correlator and step-wise power spectrum (47) can be constructed by the SFG method only if its parameters $k_{c}$ and $h$ are placed onto or above the upper line in Fig. 5. Only in this area of the $\left(k_{c}, h\right)$-plane the condition (54) holds true. Unfortunately, practically within this whole area the parameters $k_{c}$ and $h$ have values of the order of one, and, therefore, one cannot satisfy a quite strong requirement (48) in order to clearly observe a mobility edge. The only exception is a narrow vicinity of the point $\left(k_{c}=0, h=1\right)$. Remarkably, in this vicinity due to specific dependence (57) of $h_{\min }\left(k_{c}\right)$, the conditions (49) can be satisfied,

$$
0<k_{c} \ll 1-h \leqslant 1-h_{\min }\left(k_{c}\right) \approx\left(\frac{96 k_{c}}{\pi^{4}}\right)^{1 / 3} \ll 1,
$$

and, consequently, the mobility edge can be achieved. So, Eq. (58) gives us the only (perhaps, just purely theoretical) possibility to arrange a mobility edge in the transport through the $\gamma$-sequence constructed by the SFG method.

Now we analyze the consequences of the necessary conditions formulated in Sec. II. For the long-range correlator (47a) the inequalities (19) lead to the following restriction with respect to the step-parameter $h$,

$$
1-\max ^{-1}\left\{R\left(r, r^{\prime}\right)\right\} \leqslant h
$$




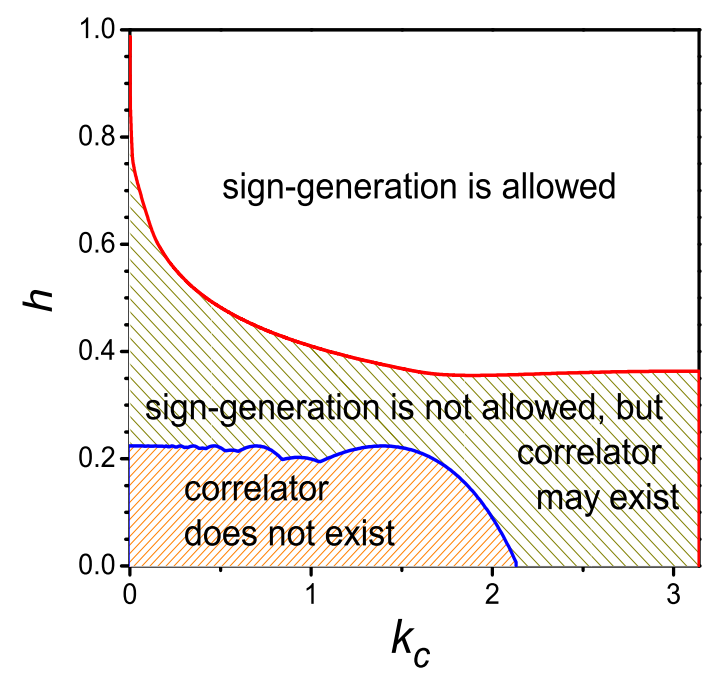

FIG. 5: (Color online) Space of control parameters $k_{c}$ and $h$. Upper curve is the dependence $h_{\min }\left(k_{c}\right)$ while low curve depicts $h_{0}\left(k_{c}\right)$. Within the lowest area in which $0 \leqslant h<h_{0}$, a dichotomic sequence $\gamma_{n}$ with the correlator $K_{\gamma, h}(r)$ does not exist. The area with $h_{\min } \leqslant h \leqslant 1$ allows for a creation of $\gamma_{n}$ with $K_{\gamma, h}(r)$ by the discussed method. In the intermediate region $h_{0} \leqslant h<h_{\text {min }}$ the SFG method does not work, and an existence of a dichotomic sequence with the step-wise spectrum remains an open problem.

at $r \neq 0, r^{\prime} \neq 0$ and $r \neq r^{\prime}$. Here we have introduced the function

$$
R\left(r, r^{\prime}\right)=\left|\frac{\sin k_{c} r^{\prime}}{k_{c} r^{\prime}} \pm \frac{\sin k_{c}\left(r-r^{\prime}\right)}{k_{c}\left(r-r^{\prime}\right)}\right| \mp \frac{\sin k_{c} r}{k_{c} r} .
$$

Since by the definition $h \geqslant 0$, the requirement (59) is meaningful only if its l.h.s. is positive. Otherwise, it is satisfied automatically. The combination of Eq. (59) with the assumption $0 \leqslant h \leqslant 1$ gives rise to the relation,

$$
h_{0} \leqslant h \leqslant 1 \text {, }
$$

where new characteristic quantity $h_{0}$ is introduced,

$$
h_{0}=1-\max ^{-1}\left\{1, \max \left\{R\left(r, r^{\prime}\right)\right\}\right\} .
$$

This function $h_{0}\left(k_{c}\right)$ is shown in Fig. 5 by low curve. Its piecewise shape is caused by the fact that different $r$ and $r^{\prime}$ contribute to $h_{0}$ within different intervals of $k_{c}$. Finally, when $k_{c}$ becomes equal or larger than $k^{*} \simeq$ $2.139 \ldots$ [see text after Eq. (46)], we have $h_{0}=0$ and the necessary conditions (61) reduce to the initial ones, $0 \leqslant h \leqslant 1$.

Thus, taking into account that the required area of the parameters $k_{c}$ and $h$ is determined by the relation, $0<$ $k_{c}<\pi, 0 \leqslant h \leqslant 1$ of the $\left(k_{c}, h\right)$-plane, one can summarize the following. A dichotomic sequence with long-range correlator and step-wise power spectrum (47) does not exist within the lowest region $0 \leqslant h<h_{0}\left(k_{c}\right)$. When $h_{0}\left(k_{c}\right) \leqslant h<h_{\min }\left(k_{c}\right)$, the dichotomic chain cannot be created by the SFG method and there is no answer whether it can be created by any other method. Finally, within the highest zone with $h_{\min }\left(k_{c}\right) \leqslant h \leqslant 1$, one can construct desired dichotomic sequences with the use of the discussed method.

\section{Predefined Intermediate Spectrum}

With the SFG method, we first generate intermediate Gaussian sequence $\beta_{n}$ and after, the dichotomic sequence $\gamma_{n}$. Above, we specified the correlator $K_{\gamma}(r)$ of a final dichotomic $\gamma$-sequence and analyzed the spectrum $\mathcal{K}_{\beta}(k)=\mathcal{S}\left\{K_{\gamma}\right\}(k)$ of the intermediate Gaussian $\beta$-chain, keeping in mind that the latter must be nonnegative, see Eqs. (35), (38).

Below, we ask question about the type of the spectrum of $\gamma_{n}$, that emerges if the intermediate Gaussian sequence $\beta_{n}$ is assumed to have given pair correlator with step-wise power spectrum of the following form,

$$
\begin{aligned}
K_{\beta}(r) & =\frac{\sin \left(k_{c} r\right)}{k_{c} r} \\
\mathcal{K}_{\beta}(k) & =\frac{\pi}{k_{c}} \Theta\left(k_{c}-|k|\right), \quad 0<k_{c} \leqslant \pi,|k| \leqslant \pi(63
\end{aligned}
$$

In accordance with the relation (33) and Fourier transforms (25), the corresponding correlator and power spectrum of the dichotomic $\gamma$-sequence read,

$$
\begin{array}{r}
K_{\gamma}(r)=\frac{2}{\pi} \arcsin \left[\frac{\sin \left(k_{c} r\right)}{k_{c} r}\right], \\
\mathcal{K}_{\gamma}(k)=\frac{2}{\pi} \sum_{r=-\infty}^{\infty} \arcsin \left[\frac{\sin \left(k_{c} r\right)}{k_{c} r}\right] \exp (-i k r) \\
=\left(1-\frac{2}{\pi}\right)+\frac{2}{k_{c}} \Theta\left(k_{c}-|k|\right) \\
+\frac{4}{\pi} \sum_{r=1}^{\infty}\left\{\arcsin \left[\frac{\sin \left(k_{c} r\right)}{k_{c} r}\right]-\frac{\sin \left(k_{c} r\right)}{k_{c} r}\right\} \cos (k r) .
\end{array}
$$

Here, last representation for the spectrum $\mathcal{K}_{\gamma}(k)$ is similar to that we have employed for the function $\mathcal{S}\{\cdot\}(k)$ in its analysis [compare with Eqs. (36) and (42)]. It is noteworthy to emphasize that the summand in the last term of this representation behaves as $2 / 3 \pi k_{c}^{3} r^{3}$ when $r \rightarrow \infty$. Hence, as above, the sum converges rapidly and uniformly. Therefore, it can be easily calculated numerically.

Fig. [6 presents the behavior of the power spectrum (64b) for dichotomic $\gamma_{n}$ in the case of the predefined step-wise profile (63b) for the spectrum of intermediate sequence $\beta_{n}$. Since $\mathcal{K}_{\gamma}(k)$ is an even function of the wave number $k$, the presentation is sufficient within $0 \leqslant k \leqslant \pi$. Assuming $k_{c}<\pi$, from Eq. (64b) and Fig. 66one can conclude: 


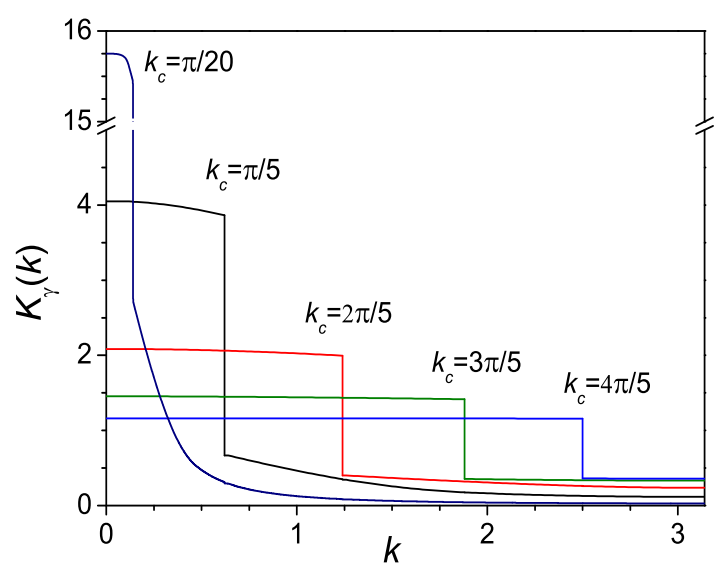

FIG. 6: (Color online) Power spectrum $\mathcal{K}_{\gamma}(k)$ vs $k$ for different values of the correlation parameter $k_{c}$.

1. The function (64b) is positive within the whole interval $|k| \leqslant \pi$. Therefore, it truly serves as a power spectrum and its inverse Fourier transform (64a) is valid correlator of the generated sequence $\gamma_{n}$;

2. Due to the second and last terms in Eq. (64b) the spectrum $\mathcal{K}_{\gamma}(k)$ decreases with an increase of $k$ for all $k_{c}$ within whole interval $(0, \pi)$;

3. The negative jump of $\mathcal{K}_{\gamma}(k)$ occurs at $k=k_{c}$, at the same point as for the jump of prescribed intermediate power spectrum $\mathcal{K}_{\beta}(k)$. The maximal and minimal values of $\mathcal{K}_{\gamma}(k)$ at the jump are defined at $k=k_{c}-0$ and $k=k_{c}+0$, respectively. The jump is exclusively formed by the second term in Eq. (64b). Therefore, its value reads

$$
\mathcal{K}_{\gamma}\left(k_{c}-0\right)-\mathcal{K}_{\gamma}\left(k_{c}+0\right)=2 / k_{c}
$$

4. The value $\mathcal{K}_{\gamma}\left(k=k_{c}-0\right)$ to the left from the jump is always positive. The value $\mathcal{K}_{\gamma}\left(k=k_{c}+0\right)$ to its right is also positive for all $k_{c}$. Their ratio $\mathcal{K}_{\gamma}\left(k_{c}-\right.$ $0) / \mathcal{K}_{\gamma}\left(k_{c}+0\right)$ being of the order of one for finite $k_{c}$, seems to slightly increase with a decrease of $k_{c}$. However, it is saturated if the inverse correlation length $k_{c}$ vanishes. Indeed, from Eq. (64b) one can easily get,

$$
\begin{aligned}
\frac{\mathcal{K}_{\gamma}\left(k_{c}-0\right)}{\mathcal{K}_{\gamma}\left(k_{c}+0\right)} & =\frac{\pi+I\left(k_{c}\right)+(\pi / 2-1) k_{c}}{I\left(k_{c}\right)+(\pi / 2-1) k_{c}} \\
& \left.\rightarrow \frac{\pi+I(0)}{I(0)}=6.0096 \ldots \text { if } k_{c} \rightarrow \emptyset 66 \mathrm{~b}\right)
\end{aligned}
$$

\section{A. Power Correlator for Dichotomic Sequence}

Here we consider an important problem of constructing a sequence with the power correlation function and 
corresponding spectrum,

$$
\begin{aligned}
K_{\gamma, p}(r)= & \delta_{r, 0}+\left(k_{c}|r|\right)^{-p}\left(1-\delta_{r, 0}\right), \\
\mathcal{K}_{\gamma, p}(k)= & 1 \\
+ & \left.k_{c}^{-p}\left\{\operatorname{Li}_{p}[\exp (i k)]+\operatorname{Li}_{p}[\exp (-i k)]\right\} 70 \mathrm{~b}\right) \\
& \quad p>0, \quad k_{c} \geqslant 1, \quad|k| \leqslant \pi .
\end{aligned}
$$

Here $p$ and $k_{c}$ are positive real numbers characterizing how fast the correlator decreases. Note that the parameter $k_{c}$ cannot be less than one since $K_{\gamma, p}(r) \leqslant 1$. The Fourier transform $\mathcal{K}_{\gamma, p}(k)$ of this correlator is expressed via the polylogarithm function $\operatorname{Li}_{q}(z)$ that is defined by

$$
\operatorname{Li}_{q}(z)=\sum_{r=1}^{\infty} \frac{z^{r}}{r^{q}}
$$

For $K_{\gamma, p}(r)$ to be the correlator of a stochastic process, it is necessary to have $\mathcal{K}_{\gamma, p}(k) \geqslant 0$ for all $k$. This condition is satisfied if and only if the following inequality is fulfilled,

$$
k_{c} \geqslant\left[-2 \operatorname{Li}_{p}(-1)\right]^{1 / p} .
$$

This result is due to the shape of spectrum $\mathcal{K}_{\gamma, p}(k)$ that monotonously decreases with an increase of $k$ within the interval $(0, \pi)$, and reaches its minimal value at $k=\pi$. The r.h.s. of the condition (172) can be calculated in limit cases,

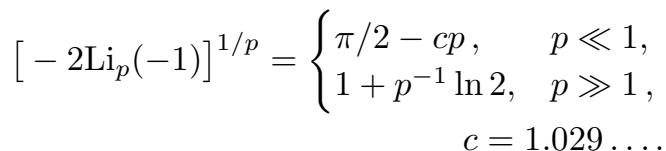

In Fig. 7 the area where power spectrum (70b) is nonnegative, is located above the dotted lowest curve.

If $K_{\gamma, p}(r)$ is the correlator of a dichotomic random sequence generated by the SFG method, then $\mathcal{S}\left\{K_{\gamma, p}\right\}(k) \geqslant 0$ for all values of $k$. Since this function, as well as $\mathcal{K}_{\gamma, p}(k)$, monotonously decreases, the only condition is required,

$$
\mathcal{S}\left\{K_{\gamma, p}\right\}(\pi) \geqslant 0 .
$$

Expanding in Eq. (36a) the sin-function into series, we get useful expression

$$
\begin{aligned}
& \mathcal{S}\left\{K_{\gamma, p}\right\}(k)=1+\sum_{l=0}^{\infty} \frac{\pi^{2 l+1}}{2^{2 l+1}(2 l+1) !} k_{c}^{-p(2 l+1)} \\
& \times\left\{\operatorname{Li}_{p(2 l+1)}[\exp (i k)]+\operatorname{Li}_{p(2 l+1)}[\exp (-i k)]\right\} .
\end{aligned}
$$

For $p \rightarrow \infty$ it can be approximately calculated as

$$
\mathcal{S}\left\{K_{\gamma, p}\right\}(k) \simeq 1+2 \sin \left(\pi k_{c}^{-p} / 2\right) \cos k .
$$

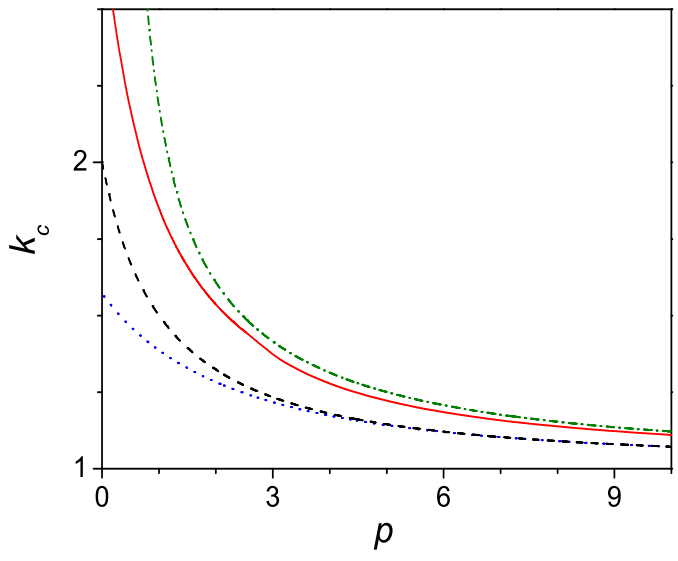

FIG. 7: (Color online) Various borders of the parameters $p$ and $k_{c}$ above which the following relations are fulfilled: (a) Eq. (72) (dotted curve), (b) Eq. (74)) (solid curve), (c) Eq. (80)) (dashed curve), (d) Eq. (84) (dash-dotted curve).

Therefore, taking into account that $k_{c} \geqslant 1$, one obtains the following asymptotic of the condition (74)

$$
k_{c} \geqslant 1+p^{-1} \ln 3 \quad \text { for } \quad p \gg 1 .
$$

The area where the discussed SFG method is applicable located in Fig. 7 above solid curve.

Now we analyze the necessary condition for existence of the power correlator (70a) for dichotomic sequence regardless of the generation method. For the sake of simplicity we consider the case $\bar{\gamma}=0$. From the inequalities (19) one can obtain the following relation,

$$
\begin{aligned}
& \max \left\{\left.|| r^{\prime}\right|^{-p} \pm\left.\left|r-r^{\prime}\right|^{-p}|\mp| r\right|^{-p}\right\} \leqslant k_{c}^{p}, \\
& r \neq 0, \quad r^{\prime} \neq 0, \quad r \neq r^{\prime} .
\end{aligned}
$$

It is easy to see that the maximum with respect to $r^{\prime}$, occurs at $r^{\prime}= \pm 1$ or $r^{\prime}=r \pm 1$. Therefore, Eq. (78) can be rewritten as

$$
\max _{r>0}\left\{1 \pm(r+1)^{-p} \mp r^{-p}\right\} \leqslant k_{c}^{p} .
$$

The last condition is equivalent to

$$
k_{c} \geqslant\left(2-2^{-p}\right)^{1 / p}= \begin{cases}2-2 p \ln ^{2} 2, & p \ll 1, \\ 1+p^{-1} \ln 2, & p \gg 1 .\end{cases}
$$

In Fig. 7 the border corresponding to this condition is designated by dashed curve. It should be noted that Eq. (80) is the necessary condition, thus, if it is met, it is still not clear whether the correlator with such values of parameters $p$ and $k_{c}$ exists.

\section{B. Predefined Intermediate Power Correlator}

We have found that the dichotomic sequence $\gamma_{n}$ with the power correlator (70a) can be constructed by the SFG 
method for some values of parameters $p$ and $k_{c}$ that meet the condition (74). Now let us take the intermediate Gaussian sequence $\beta_{n}$ prescribed to have the power correlator and corresponding spectrum

$$
\begin{aligned}
K_{\beta, p}(r)= & \delta_{r, 0}+\left(k_{c}|r|\right)^{-p}\left(1-\delta_{r, 0}\right) \\
\mathcal{K}_{\beta, p}(k)= & 1 \\
+ & k_{c}^{-p}\left\{\operatorname{Li}_{p}[\exp (i k)]+\operatorname{Li}_{p}[\exp (-i k)]\right\} \\
& p>0, \quad k_{c} \geqslant 1, \quad|k| \leqslant \pi
\end{aligned}
$$

Evidently, the condition (72) is implied to be met. In accordance with the relation (33) and Fourier transforms (25), the correlator and power spectrum of the generated dichotomic $\gamma$-sequence are described as

$$
\begin{gathered}
K_{\gamma, p}(r)=\delta_{r, 0}+\frac{2}{\pi} \arcsin \left[\left(k_{c}|r|\right)^{-p}\right]\left(1-\delta_{r, 0}\right), \\
\mathcal{K}_{\gamma, p}(k)=1+\frac{4}{\pi} \sum_{r=1}^{\infty} \arcsin \left[\left(k_{c} r\right)^{-p}\right] \cos (k r) .
\end{gathered}
$$

We can assert that since Eq. (72) is satisfied, i.e. the spectrum (81b) of the intermediate $\beta$-sequence is nonnegative, then the spectrum $(82 \mathrm{~b}$ ) of the generated dichotomic $\gamma_{n}$ is also non-negative.

When $|r| \rightarrow \infty$, the correlator (82a) tends to zero in accordance with the following asymptotic

$$
K_{\gamma, p}(r) \simeq\left(k_{c}^{\prime}|r|\right)^{-p}, \quad k_{c}^{\prime}=k_{c}(\pi / 2)^{1 / p} .
$$

As was shown, the allowed values of $k_{c}$ are expressed by Eq. (72). Therefore, the scaling parameter $k_{c}^{\prime}$ should satisfy the condition

$$
k_{c}^{\prime} \geqslant\left[-\pi \operatorname{Li}_{p}(-1)\right]^{1 / p} .
$$

This condition for possible values of $k_{c}^{\prime}$ and $p$ is met in the area above the dash-dotted curve in Fig. 7.

Thus, the mapping of the gaussian sequence with the power correlation function 81a into the binary sequence result in the same power for the decrease of the final correlator (82a) expressed by Eq. (83). However, such a behavior of the final correlator occurs only asymptotically, for sufficiently large values of $|r|$.

\section{CONCLUSION}

First, we would like to emphasize the following point that was briefly mentioned in the beginning. Our study of the correlation properties of a random dichotomic sequence $\gamma_{n}$ is based on the example (1) in which two elements are " -1 " and " 1 ". On the other hand, there is a simple correspondence between this chain and a dichotomic sequence $\varepsilon(n)$ consisting of two arbitrary symbols $\varepsilon_{0}$ and $\varepsilon_{1}$,

$$
\varepsilon(n)=\left\{\varepsilon_{0}, \varepsilon_{1}\right\}, \quad n \in \mathbf{Z}=\ldots,-2,-1,0,1,2, \ldots
$$

The correspondence is expressed by the linear relationship,

$$
\varepsilon(n)=\frac{\varepsilon_{0}+\varepsilon_{1}}{2} \mp \frac{\varepsilon_{0}-\varepsilon_{1}}{2} \gamma_{n} .
$$

The choice of the sign is not important. It derermines only into what symbol, $\varepsilon_{0}$ or $\varepsilon_{1}$, the initial values " -1 " and "1" are converted.

In accordance with Eq. (86) and due to specific properties (30), (3) of the $\gamma$-sequence, the connection between the mean values and variances is as follows,

$$
\begin{aligned}
& \varepsilon^{2}(n)=\frac{\varepsilon_{0}^{2}+\varepsilon_{1}^{2}}{2} \mp \frac{\varepsilon_{0}^{2}-\varepsilon_{1}^{2}}{2} \gamma_{n} \\
& \bar{\varepsilon}=\frac{\varepsilon_{0}+\varepsilon_{1}}{2} \mp \frac{\varepsilon_{0}-\varepsilon_{1}}{2} \bar{\gamma} \\
& C_{\varepsilon}(0) \equiv \overline{\varepsilon^{2}(n)}-\bar{\varepsilon}^{2}=\frac{\left(\varepsilon_{0}-\varepsilon_{1}\right)^{2}}{4} C_{\gamma}(0) .
\end{aligned}
$$

Analogously, the two-point correlation function $C_{\varepsilon}(r)$ of the $\varepsilon$-chain is associated with the binary correlation function $C_{\gamma}(r)$ of the sequence $\gamma_{n}$ as follows

$$
C_{\varepsilon}(r) \equiv \overline{\varepsilon(n) \varepsilon(n+r)}-\bar{\varepsilon}^{2}=\frac{\left(\varepsilon_{0}-\varepsilon_{1}\right)^{2}}{4} C_{\gamma}(r) .
$$

The comparison of Eqs. (87c) and (88) makes obvious the equality between the normalized correlators $K_{\varepsilon}(r)$ and $K_{\gamma}(r)$,

$$
K_{\varepsilon}(r) \equiv C_{\varepsilon}(r) / C_{\varepsilon}(0)=C_{\gamma}(r) / C_{\gamma}(0) \equiv K_{\gamma}(r) .
$$

Thus, our analysis is valid for any dichotomic sequence.

Our results can be summarized as follows. We have shown that the statistical properties of random dichotomic sequences are principally different from those known for sequences with a continuous distribution of their elements. We were able to find analytically the conditions (19) that can be used to know whether a binary sequence can have the desired pair correlator. Note that these two conditions are necessary only.

Another important restriction is due to the inequality (39) derived under quite general assumptions. We have shown that even in the well known case of an exponential decay of correlations, there are no binary sequences that can be created with the SFG method, unless the decay is sufficiently strong. This fact is very important in view of many applications.

Our specific interest was in a possibility to create, with the considered method, the binary sequences with longrange correlations described by Eqs. (41a) and (41b). We have analytically found that the function (41a) can not be a pair correlator of any binary sequence. We have also examined a more general correlation function [see Eq. (47)] that corresponds to the generalization of the step-wise power spectrum. Our extensive examination of the signum-function method, applied to this correlation function, has revealed the regions of parameters $k_{c}$ and $h$ 
for which the pair correlator can emerge in a binary sequence. Correspondingly, we identified the regions where such a pair correlator can not appear in binary sequences. For other values of the control parameters we can not give definite answer, therefore, a further study is needed.

Finally, we analyzed an important case of the power decay of the pair correlator. Recently, the problem of the generation of random processes with power correlations has attracted much attention in the literature. Analyzing such correlators, we have found that the SFG method in principle allows to construct binary sequences with these correlators, however, with some restrictions on the values of parameters in Eq. (70).

\section{ACKNOWLEDGMENTS}

This work was partly supported by the CONACYT (México) grant No 43730.

\section{APPENDIX A: PROBABILITY DENSITY OF $\beta_{n}$}

The standard way to derive the probability density $\rho_{B}(\beta)$ of the random process $\beta_{n}$ is due to its characteristic function $\varphi_{B}(t)$ defined by

$$
\varphi_{B}(t) \equiv \overline{\exp \left[i t \beta_{n}\right]}=\int_{-\infty}^{\infty} d \beta \rho_{B}(\beta) \exp (i t \beta) .
$$

From the last equality in this definition it immediately follows that the probability density $\rho_{B}(\beta)$ is the Fourier transform of $\varphi_{B}(t)$,

$$
\rho_{B}(\beta)=\frac{1}{2 \pi} \int_{-\infty}^{\infty} d t \varphi_{B}(t) \exp (-i t \beta) .
$$

To start with, we substitute the explicit expression (20b) for $\beta_{n}$ into the definition (A1) for characteristic function $\varphi_{B}(t)$. Then, we rewrite the result as an infinite product of exponential functions and take into account the statistical independence of uncorrelated random variables $\alpha_{n}$. This procedure yields

$$
\overline{\exp \left[i t \beta_{n}\right]}=\exp (i t \bar{\beta}) \prod_{n^{\prime}=-\infty}^{\infty} \overline{\exp \left[i t G\left(n-n^{\prime}\right) \alpha_{n^{\prime}}\right]} \text {. }
$$

In accordance with the Gaussian distribution (21b) of $\alpha_{n}$, its characteristic function is

$$
\begin{aligned}
\varphi_{A}(\tau) \equiv \overline{\exp \left(i \tau \alpha_{n}\right)} & \equiv \int_{-\infty}^{\infty} d \alpha \rho_{A}(\alpha) \exp (i \tau \alpha) \\
& =\exp \left(-\tau^{2} / 2\right) .
\end{aligned}
$$

The use of Eqs. A3 , (A4) with $\tau=t G\left(n-n^{\prime}\right)$, and the normalization condition (24) results in

$$
\varphi_{B}(t)=\exp \left(i \bar{\beta} t-t^{2} / 2\right) .
$$

As is known, this characteristic function corresponds to the Gaussian probability density (28). One can confirm this fact by a direct evaluation of the integral in Eq. (A2).

\section{APPENDIX B: PAIR CORRELATOR OF $\gamma_{n}$}

Let us derive the pair correlator $\overline{\gamma_{n} \gamma_{n+r}}$. Employing the standard integral presentation for the signum function,

$$
\operatorname{sign}(z)=\frac{1}{\pi} \int_{-\infty}^{\infty} d x \frac{\sin (z x)}{x}
$$

and Eq. (A4), we arrive, in a manner similar to the calculation of the characteristic function $\varphi_{B}(t)$ in Appendix $\mathrm{A}$. at the expression,

$$
\begin{aligned}
& \overline{\gamma_{n} \gamma_{n+r}}=\mathcal{J}\left(K_{\beta}(r), \bar{\beta}\right) \\
& =\frac{2}{\pi^{2}} \int_{0}^{\infty} \frac{d x_{1}}{x_{1}} \int_{0}^{\infty} \frac{d x_{2}}{x_{2}} \exp \left(-\frac{x_{1}^{2}+x_{2}^{2}}{2}\right) \\
& \times \sum_{s=-1,1} s \exp \left[s K_{\beta}(r) x_{1} x_{2}\right] \cos \left[\bar{\beta}\left(x_{1}-s x_{2}\right)\right]
\end{aligned}
$$

Thus, we have reduced the problem to the derivation of $\mathcal{J}(K, \beta)$. To solve it, we obtain the derivative of $\mathcal{J}(K, \beta)$ with respect to $K$. After some simplifications one gets,

$$
\begin{aligned}
\frac{\partial}{\partial K} \mathcal{J}(K, \beta)= & \frac{1}{\pi^{2}} \int_{0}^{\infty} d x_{1} \int_{-\infty}^{\infty} d x_{2} \exp \left(-\frac{x_{1}^{2}+x_{2}^{2}}{2}\right) \\
& \times \exp \left[K x_{1} x_{2}\right] \sum_{t=-1,1} \exp \left[i t \beta\left(x_{1}-x_{2}\right)\right] .
\end{aligned}
$$

To proceed, we write down the following relation that is valid for arbitrary real quantities $a$ and $b$,

$$
\begin{gathered}
\int_{-\infty}^{\infty} d x \exp \left(-x^{2} / 2\right) \exp (a x) \exp (i b x) \\
\quad=\sqrt{2 \pi} \exp \left[\left(a^{2}-b^{2}\right) / 2\right] \exp (i a b) .
\end{gathered}
$$

Using Eq. (B3) with $a=K x_{1}$ and $b=-t \beta$ we integrate over $x_{2}$ and make further simplifications,

$$
\begin{aligned}
\frac{\partial}{\partial K} \mathcal{J}(K, \beta)=\frac{\sqrt{2 \pi}}{\pi^{2}} \int_{-\infty}^{\infty} d x_{1} \exp \left[-x_{1}^{2}\left(1-K^{2}\right) / 2\right] \\
\times \exp \left(-\beta^{2} / 2\right) \exp \left[i \beta(1-K) x_{1}\right] .
\end{aligned}
$$

Now we change the integration variable $x_{1}$,

$$
x_{1}^{\prime}=x_{1} \sqrt{1-K^{2}} .
$$

Then, applying Eq. (B3) with

$$
a=0, \quad b=\beta \sqrt{\frac{1-K}{1+K}},
$$

we perform the integration over $x_{1}$ that gives rise to the expression

$$
\frac{\partial}{\partial K} \mathcal{J}(K, \beta)=\frac{2}{\pi \sqrt{1-K^{2}}} \exp \left(-\frac{\beta^{2}}{1+K}\right) .
$$


The general solution of Eq. (B6) is

$$
\begin{aligned}
\mathcal{J}(K, \beta) & =\mathcal{J}(0, \beta) \\
& +\frac{2}{\pi} \int_{0}^{K} \frac{d x}{\sqrt{1-x^{2}}} \exp \left(-\frac{\beta^{2}}{1+x}\right) .
\end{aligned}
$$

It should be noted that Eq. (B7) can be also obtained by means of the two-point probability density that for the correlated Gaussian sequence $\beta_{n}$ with the correlator $K_{\beta}(r)$ is defined by

$$
\begin{aligned}
& \rho_{B}\left(\beta_{n}=\beta, \beta_{n+r}=\beta^{\prime}\right) \\
= & \frac{1}{2 \pi \sqrt{1-K_{\beta}^{2}(r)}} \exp \left\{-\frac{\beta^{2}+\beta^{\prime 2}-2 K_{\beta}(r) \beta \beta^{\prime}}{2\left[1-K_{\beta}^{2}(r)\right]}\right\} .
\end{aligned}
$$

The last step we should take, is to calculate $\mathcal{J}(0, \beta)$.
It can be directly obtained from Eq. (B2),

$$
\mathcal{J}(0, \bar{\beta})=\left[\frac{2}{\pi} \int_{0}^{\infty} d x \frac{\sin (\bar{\beta} x)}{x} \exp \left(-x^{2} / 2\right)\right]^{2}=\bar{\gamma}^{2}
$$

This result can be easily explained. Indeed, the condition $K_{\beta}(r)=0$ implies that the correlations between $\beta_{n}$ and $\beta_{n+r}$ disappear, hence, the correlations between $\gamma_{n}$ and $\gamma_{n+r}$ are absent as well.

As a result of these calculations, we finally get

$\mathcal{J}\left(K_{\beta}(r), \bar{\beta}\right)=\bar{\gamma}^{2}+\frac{2}{\pi} \int_{0}^{K_{\beta}(r)} \frac{d x}{\sqrt{1-x^{2}}} \exp \left(-\frac{\bar{\beta}^{2}}{1+x}\right)$

This expression provides Eq. (32).
[1] I. M. Lifshits, S. A. Gredeskul and L. A. Pastur, Introduction to the Theory of Disordered Systems ( New York: Wiley, 1988)

[2] N. M. Makarov, I. V. Yurkevich, Zh. Eksp. Teor. Fiz. 96 1106 (1989) [Sov. Phys. JETP 69628 (1989)]; V. D. Freilikher, N. M. Makarov, I. V. Yurkevich, Phys. Rev. B 41, 8033 (1990).

[3] M. Griniasty, S. Fishman, Phys. Rev. Lett. 60, 1334 (1988).

[4] N. M. Makarov, Lectures on Spectral and Transport Properties of One-Dimensional Disordered Conductors http://www.ifuap.buap.mx/virtual/page_vir.html, 1999.

[5] F. Moura and M. L. Lyra, Phys. Rev. Lett. 81, 3735 (1998).

[6] F. M. Izrailev, A. A. Krokhin, Phys. Rev. Lett. 82, 4062 (1999); A. A. Krokhin, F. M. Izrailev, Ann. Phys. (Leipzig) SI-8, 153 (1999).

[7] F. M. Izrailev, N. M. Makarov, J. Phys. A: Math. Gen. 38, 10613 (2005).

[8] S. O. Rice, Bell Syst. Tech. J. 23, 282 (1944); S. O. Rice, in Selected Papers on Noise and Stochastic Processes, ed. by N. Wax (Dover, New York, 1954) p. 180.

[9] D. Saupe, in The Science of Fractal Images, ed. by H.O. Peitgen and D. Saupe (Springer, New York, 1988); J. Feder, Fractals (Plenum Press, New York, 1988).

[10] C.-K. Peng et al., Phys. Rev. A 44, 2239 (1991).
[11] S. Prakash et al., Phys. Rev. A 46, R1724 (1992).

[12] C. S. West, K. A. O'Donnell, J. Opt. Soc. Am. A 12, 390 (1995).

[13] A. Czirok, R. N. Mantegna, S. Havlin, H. E. Stanley, Phys. Rev. E 52, 446 (1995).

[14] F. M. Izrailev, N. M. Makarov, Opt. Lett. 26, 1604 (2001); F. M. Izrailev, N. M. Makarov, Phys. Rev. B 67, 113402 (2003).

[15] F. M. Izrailev, N. M. Makarov, Appl. Phys. Lett. 84, 5150 (2004).

[16] F. M. Izrailev, A. A. Krokhin, N. M. Makarov, and O.V.Usatenko, Phys. Rev. E. 76027701 (2007).

[17] S. S. Melnyk, O. V. Usatenko, V. A. Yampolśkii, and V. A. Golick, Phys. Rev. E, 72, 026140 (2005).

[18] S. S. Melnyk, O. V. Usatenko, and V. A. Yampolśkii, Physica A, 361, 405 (2006).

[19] V. A. Yampolśkii, S. Savelév, O. V. Usatenko, S. S. Melńik, F. V. Kusmartsev, A. A. Krokhin, and F. Nori, Phys. Rev. B, 75, 014527 (2007).

[20] P. Carpena, P. Bernaola-Galv́an, P. Ch. Ivanov, H. E. Stanley, Nature 418, 955 (2002); Nature 421, 764 (2003).

[21] A. Erdelyi, Higher Transcendental Functions, V. 2, McGraw-Hill, 1953.

[22] F. M. Izrailev, A. A. Krokhin, N. M. Makarov, S. S. Melnyk, O. V. Usatenko, and V. A. Yampol'skii, Physica A 372, 279 (2006). 